\title{
Task-switch costs subsequent to cue-only trials
}

Rachel Swainson*, Douglas Martin, Laura Prosser

School of Psychology, University of Aberdeen AB24 2UB

Running head: Switch costs after cue-only trials

* Corresponding author. Address: School of Psychology, William Guild Building, University of Aberdeen AB24 2UB. Tel: 01224 273918. Email: r.swainson@abdn.ac.uk

\section{Acknowledgements}

The authors would like to thank the following: Fiona Carr, Carmen Horne and Brigitta Toth, for assistance with data collection; and the School of Psychology, University of Aberdeen, for contributing funding for participant payments. 


\begin{abstract}
There is abundant evidence that there is a performance cost associated with switching between tasks. This "switch cost" has been postulated to be driven by task-performance on the preceding trial, but recent research challenges any necessary role of previous taskperformance in driving the cost. Across three experiments, we investigated whether it is difficult to switch from a task that was prepared but never performed. We replicated the finding of a switch cost following cue-only trials (involving no task-performance) whilst controlling for a potential cue-switching confound. This cost was larger than that following completed trials when preparation interval was short $(300 \mathrm{msec})$ and it reduced significantly with a longer preparation interval $(1000 \mathrm{msec})$ on the current trial. We also found that preparing only to attend to a particular visual dimension (colour or shape) was sufficient to drive a significant subsequent switch cost which appeared to be residual in nature; we speculate that this cost may reflect the persistence of unfulfilled task-intentions and/or a strategic slowing when consecutive intentions conflict.
\end{abstract}

Keywords: task-switching; subsequent switch cost; preparation; stimulus-response mappings 
Human behaviour is massively flexible. We can select innumerable actions on the basis of a huge variety of internal and external information, and respond in very different ways to the same stimulus depending upon circumstances (e.g. kicking a ball in football, versus throwing it in netball). These actions are rarely completely isolated, often occurring within an ongoing sequence of activity, and necessitating rapid switches between different behaviour-guiding rules. Not all of the actions which we intend to perform are eventually executed - we may be interrupted or circumstances may change. The research presented here investigates the impact upon subsequent performance of forming an intention which remains unfilled.

One way of studying the dynamics of behavioural control is by using the taskswitching procedure, where different rules for selecting a response on the basis of a stimulus (“tasks") must be used on different trials, often according to a cue stimulus (see reviews by by Kiesel et al., 2010; Monsell, 2003; Vandierendonck, Liefooghe, \& Verbruggen, 2010). A key finding in task-switching studies is that of the "switch cost", whereby performance speed and/or accuracy is poorer when switching between different tasks than when repeating the same task on consecutive trials. Whilst there is continuing debate over the precise nature of the switch cost itself, at a minimum its existence would seem to indicate a difference in the state of readiness to perform a particular task depending upon whether the same or a different task had been performed previously: i.e., immediately after performing task A, we are usually more ready to perform task A than to perform task B. In the current paper we explore what it is that needs to happen in order that this differential state of readiness is set up - in other words, we investigate the necessary conditions for a "subsequent switch cost" to exist. 
It is very well established that performing a particular task usually results in a switch cost measured on the subsequent trial, but it is not yet clear which stages of processing (e.g. task-preparation, target-judgement, response-selection, response-execution) are necessary to trigger a subsequent switch cost. In a number of recent studies (e.g. Los \& Van der Burg, 2010; Philipp, Jolicoeur, Falkenstein, \& Koch, 2007; Schuch \& Koch, 2003; Swainson \& Martin, 2013; Verbruggen, Liefooghe, \& Vandierendonck, 2006), this issue has been investigated by amending the task-switching procedure such that on a proportion of trials task-processing is enabled only up to a particular stage of interest; the question is whether a switch cost will be present on the following trial. For example, on a particular trial a response might be selected but not executed; any switch cost present on the following trial could not logically be driven by the persisting effects of response-execution, but must instead be due the effects of an earlier stage such as response-selection or task-preparation.

A seminal paper on this topic by Schuch and Koch (2003) concluded that responseselection was the critical stage of processing necessary to produce a subsequent switch cost. The design of that study included "no-go" trials, on which presentation of a pre-target taskcue enabled task-preparation to take place but on which response-selection was either unnecessary (because a "no-go" stimulus was presented concurrently with the target, instructing the participant not to respond - Expt. 1a) or impossible (because a stimulus that was not mapped to any response was presented in place of the target - Expt. 1b). Schuch and Koch reported that there were no switch costs (and also no evidence of "backward inhibition" on switch trials - i.e., no additional cost for an ABA sequence compared to a $\mathrm{CBA}$ sequence, thought to indicate that the switched-from task is inhibited in order reduce competition with the switched-to task) following no-go trials ${ }^{1}$ and proposed that response-selection was the

\footnotetext{
${ }^{1}$ In Expt. 1b there was in fact an overall switch cost in errors which was not significantly altered according to whether the preceding trial was a go or a no-go; it is not clear whether the cost itself would have been significant specifically on trials preceded by a no-go trial.
} 
critical stage at which a task was selected, via a competitive inhibitory process, for the control of behaviour. They concluded that whilst task-preparation could activate alternative tasks, possibly including their stimulus-response mappings, it would not select one task in favour of other available tasks and would therefore produce no switch cost on the next trial. Subsequent studies using versions of the "no-go" method have added to the evidence that preparation alone does not cause a switch cost to be present on the next trial (Astle, Jackson, \& Swainson, 2006; Los \& Van der Burg, 2010) and that instead what is critical is that the prepared task is used to process a target stimulus for the selection of either a task-appropriate overt response (Philipp et al., 2007; Verbruggen et al., 2006) or a task-appropriate covert judgement (e.g. the target's particular colour or shape; Swainson \& Martin, 2013).

A challenge to the idea that preparation alone is insufficient to trigger subsequent switch costs came from Lenartowicz, Yeung and Cohen (2011; see also Brass \& von Cramon, 2004, and Desmet, Fias, \& Brass, 2012). Lenartowicz et al. compared two methods of terminating performance at the task-preparation stage. They found that switch costs were absent following no-go trials (on which a neutral stimulus, not associated with any response, was presented instead of a target) but present following "cue-only" trials that were simply truncated after preparation (negating the need for a no-go stimulus). To explain this difference in results, the authors suggested that no-go stimuli may have the effect of clearing working memory, preventing the effects of the task-preparation that had already taken place on that trial from impacting upon performance on the subsequent trial. If this is the case, it would mean that studies using no-go stimuli have tended to be blind to the potential effects of task-preparation on subsequent trials.

The idea that task-preparation can be sufficient to produce a subsequent switch cost is strikingly different from the proposal put forward by Schuch and Koch (2003), according to which preparation should not be able to trigger a subsequent switch cost as it involves no 
competitive task-selection process (see also Los \& Van der Burg, 2010). In the current paper we aimed to explore in more detail the nature of the cue-driven subsequent switch cost described by Lenartowicz et al. (2011).

We began by aiming to replicate the finding that switch costs can follow cue-only trials, both with a different pair of tasks and whilst controlling for a potential confound present in the Lenartowicz et al. study. This potential confound stemmed from their use of only one cue to indicate each task - i.e., every task-switch was accompanied by a cue-switch and every task-repeat by a cue-repeat. Cue-switching is associated with performance costs independently of the need for task-switching (Logan \& Bundesen, 2003; Mayr \& Kliegl, 2003), so it is possible that the switch costs measured by Lenartowicz et al. following cueonly trials were at least partly cue-switch costs. Because two other studies using a slightly different "double-cue" design to control for cue-switching (Brass \& von Cramon, 2004, and Desmet et al., 2012) had both shown a cost when the tasks indicated by the first and second cues differed, we anticipated that we too would observe subsequent switch costs following cue-only trials with our design.

We also wished to determine whether a switch cost following cue-only trials was the same type of switch cost as that which follows completed trials and we did that by examining the effect of preparation time on the current trial. It is well established that a part of the switch cost, referred to here as the "reducible" switch cost, can be reduced by increasing the time available for preparation on the current trial (Meiran, 1996; Rogers \& Monsell, 1995): this preparation-based reduction is known as the "reduction in switch cost" (RISC) effect (Monsell \& Mizon, 2006). Another part, known as the "residual" switch cost (Rogers \& Monsell, 1995), remains in most studies in the face of a substantial preparation interval, and is therefore apparently only able to be overcome by using the task to process a target-stimulus according to the current task rule (henceforth, task-performance). It may be that there is a 
parallel distinction between preparation and performance in terms of which processes on the preceding trial are responsible for these two types of cost being present on the current trial, such that previous preparation triggers the reducible switch cost whereas previous performance triggers the residual switch cost (c.f. Lenartowicz et al., 2011). As pointed out by Lenartowicz et al., the results of Schuch and Koch (2003) did not quite fit this pattern: in that study, whilst the residual switch cost was absent following no-go trials, the reducible part of the switch cost was also absent, even though preparation presumably took place on the preceding no-go trial. Lenartowicz et al. went on to show that a preparation-driven reducible switch cost could in fact be measured if cue-only trials were used instead of no-go trials, and we expect to find the same reducible cost following cue-only trials in the current study. In contrast, we have no strong reason to anticipate that a subsequent residual switch cost would be triggered by preparation alone. Lenartowicz et al. found an RT switch cost of $25 \mathrm{msec}$ on long-preparation (1250 msec) trials following long-preparation cue-only trials, but did not test it specifically for significance. Brass and von Cramon (2004) and Desmet et al. (2012, Experiment 2, p. 1183) did find significant RT switch costs with an $800 \mathrm{msec}$ preparation interval on double-cue trials; however, it is not certain that $800 \mathrm{msec}$ would have been sufficient to allow advance preparation to be completed, especially with two arbitrary cues mapping to each task. In the data presented here, we examine whether there is convincing evidence of a residual, as well as a reducible, switch cost following trials which involve taskpreparation but not task-performance.

A final aim was to investigate exactly what has to be prepared on a cue-only trial in order for there to be an impact on the subsequent trial in terms of a switch cost. Specifically, we hypothesised that the reducible part of the switch cost might be associated with the preparation a specific task's stimulus-response mappings. In Experiment 3, no stimulusresponse mappings were specified for either of the tasks: pre-target cues indicated only 
which stimulus dimension (colour or shape) of the upcoming target was relevant, with a posttarget screen indicating how to select a response on each trial. We expected that the reducible part of the switch cost would be absent subsequent to cue-only trials in this experiment. In addition, if Schuch and Koch (2003) were correct in proposing that only using a task to select an appropriate response should result in a subsequent residual switch cost, we should also find no residual switch cost following cue-only trials in Experiment 3.

To summarise, the aims of this study were threefold: 1) to confirm that switch costs exist subsequent to cue-only trials when cue-switching is controlled; 2) to examine whether such costs are reduced by, and/or persist despite, preparation on the current trial; and 3) to investigate whether any switch costs subsequent to a cue-only trial depend upon stimulusresponse mappings appropriate to the cued task having been prepared.

\section{Experiment 1}

In this experiment we aimed to find out whether the finding of significant switch costs following cue-only trials (that is, trials truncated after task-cueing and before target onset; Lenartowicz et al., 2011) could be replicated using a different pair of tasks (the colour and shape tasks of Swainson and Martin, 2013) and controlling for a potential cue-switching confound by switching cues on every trial. Each task involved the use of learned stimulusresponse mappings ${ }^{2}$ which were consistent across a testing session.

\footnotetext{
${ }^{2}$ Strictly speaking, these might better be termed "feature-response mappings" as it was the route from feature, e.g. blue, rather than stimulus, e.g. blue circle, that participants were instructed to prepare; however, we use the term "stimulus-response mappings" as it is much more widely used in the literature to describe the set of links between target stimuli and responses.
} 
Preparation intervals (cue-target intervals on completed trials; cue-cue intervals on cue-only trials) were $1000 \mathrm{msec}$ on the majority of trials so that on cue-only trials there would be sufficient time to prepare for the cued task; this was important because it was the effects of that preparation on subsequent trials that we wished to examine. A few trials with $300 \mathrm{msec}$ preparation intervals were included within each block both to encourage advance preparation (Altmann, 2004) and to allow us to test for its effectiveness in the form of a RISC effect - i.e., a significant reduction in switch cost as preparation time on the current trial is increased.

\section{Method}

Participants. Thirty-eight participants (27 female) were tested in return for course credit or for $£ 5$ reimbursement for their time. The age range was 17-42 years (median 21 years). The experiment was passed by the Ethics Committee of the School of Psychology, University of Aberdeen, and complied with APA ethical standards.

Materials. The study was run on PCs running E-Prime 2.0 software (Psychology Software Tools, Inc., http://www.pstnet.com), with button-boxes for responses (Cedrus Corporation, 2003). Participants used their left and right index fingers to press horizontallyadjacent buttons. Each target stimulus consisted of a coloured shape $(2.5 \mathrm{cms}$ wide) presented centrally on a black background: a circle, star, triangle or square coloured red, blue, yellow or green. For each task there were two alternative task-cueing words (presented in white, centrally on a black background): "COLOUR" and "HUE" cued the colour task; "SHAPE" and "FORM" cued the shape task. Figure 1 shows example stimuli and trial events. 
Procedure. Participants were assigned left-index-finger responses for two colours and two shapes, and right-index-finger responses for the remaining two colours and two shapes. These mappings were presented on-screen as a reminder before every block of trials and were counterbalanced across participants. There were several stages of practice, beginning with 10-trial blocks and with a 1000-msec preparation interval on all trials; via these practice blocks we introduced the different features of the design one at a time, building up to the full design in the final practice block. In the first two practice blocks, participants practised each task separately with all trials being "completed" (i.e., including the presentation of a target stimulus and requiring response-execution). In the third practice block, the colour and shape tasks were randomly intermixed. In the fourth practice block, $30 \%$ trials were truncated after the cue-target interval (cue-only trials). Participants were informed that on some trials no target would be presented and that every target should only be processed according to the cue which immediately preceded it. In the fifth practice block we introduced mini-blocks of trials with 300 -msec preparation intervals; this final practice block was identical to the experimental blocks, described next.

Experimental blocks (and the fifth practice block) were 35 trials long. Most of the trials presented in each experimental block (24/35) used a 1000 msec preparation interval; these will be described first. Trials with a $1000-\mathrm{msec}$ preparation interval began with presentation of the cue (e.g. the word "COLOUR") for $100 \mathrm{msec}$ and then a post-cue blank of 900 msec; thus, the interval between onset of the cue and onset of the target on completed trials, or onset of the next trial's cue on cue-only trials, was 1000 msec. Removing the cue prior to target onset was intended to encourage rapid task-preparation. Seventy percent of trials with a 1000 msec preparation interval were "completed" trials; on these, the target stimulus (e.g. a red circle) was presented at the end of the preparation interval for $200 \mathrm{msec}$, followed by a blank screen for $1200 \mathrm{msec}$, giving a total trial length for completed trials of 
$2400 \mathrm{msec}$. The time-limit for responding was $1200 \mathrm{msec}$ from the onset of the target. The remaining $30 \%$ of trials with a $1000 \mathrm{msec}$ preparation interval were "cue-only" trials; these trials ended immediately after the preparation interval and were followed immediately by the cue for the next trial; total trial length for cue-only trials was $1000 \mathrm{msec}$. Cue-only trials never occurred consecutively. Task was selected at random on each trial, resulting in $50 \%$ switch trials and $50 \%$ repetition trials overall.

On some trials a preparation interval of $300 \mathrm{msec}$ was used (100 msec cue plus 200 msec post-cue blank); none of these trials was truncated. These trials occurred in two "miniblocks" of five trials at the beginning, middle or end of the first half of each block and six trials at the beginning, middle or end of the second half of each block (Astle et al., 2006; Swainson \& Martin, 2013). The post-target blank duration on these trials was $1900 \mathrm{msec}$, making the total trial length equal to that of $1000 \mathrm{msec}$-preparation completed trials.

Cues switched on every trial to avoid confounding cue-switching with task-switching. Stimulus features (e.g. "red" and "star") for the current trial (n) never matched those of the most recently presented target (trial $n-1$ following completed trials and trial $n-2$ following cue-only trials) to ensure that any switch costs found would be at task-level rather than feature-level. In order to prevent a bias towards response-switching (versus responserepetition) across trials, which may otherwise have resulted from preventing featurerepetitions, the response for each trial was selected first (at random) and then an appropriate stimulus feature mapping to that response chosen. This was done independently for each stimulus dimension (colour and shape), so response-congruity was also unbiased.

On completed trials without a correct response, "INCORRECT" or "TOO SLOW" was presented in magenta for $500 \mathrm{msec}$, followed by a $1000 \mathrm{msec}$ blank. 
There were 32 experimental blocks, with a rest break after each. Participants were asked to respond quickly and accurately, and to try to use the cue to improve performance by preparing for the appropriate task.

Design \& Analysis. The design was entirely within-subjects. The independent variables were preparation interval (300 $\mathrm{msec}, 1000 \mathrm{msec})$, preceding trial completion (completed, cue-only) and transition (repeat, switch). However, these could not be fully orthogonally combined as there were no cue-only trials with a $300 \mathrm{msec}$ preparation interval. Therefore two analyses were carried out (by repeated-measures ANOVA). First, we investigated whether the preceding trial type affected switch costs by analysing the effects of preceding trial completion and transition on 1000-msec preparation interval trials only. Second, we assessed whether the RISC effect was present by looking specifically for an interaction between preparation interval and transition, on trials following completed trials only. The dependent variables were mean response time (RT) on correct trials and mean percentage errors (i.e., percentage of trials within a cell which were responded to with the wrong response), both measured only on completed trials. All statistical tests were 2-tailed, with an $\alpha$-level of 0.05 .

Means (and standard deviations) of RT and percentage error across participants in each condition are shown in Table 1. A measure of RT and percentage error switch cost (switch - repeat) within each available combination of preceding trial completion and preparation interval was calculated for each participant; the mean switch cost across participants in each condition is shown together with its $95 \%$ confidence interval (CI) in Figure 2. N.B. A switch cost with a $95 \%$ CI which does not include the value zero is equivalent to a significant [switch - repeat] difference in a two-tailed $t$-test. 
A final analysis looked within the RT data for evidence that the same preparatory task-switching process might underlie both the RISC effect and the switch cost that we predicted would be apparent subsequent to cue-only trials. We correlated individual participants' RISC effect scores ([switch cost on trials with a $300 \mathrm{msec}$ preparation interval, following completed trials] - [switch cost on trials with a $1000 \mathrm{msec}$ preparation interval, following completed trials]), with their preparation-driven switch cost scores (switch cost following cue-only trials, all with a long preparation interval), using the Pearson productmoment correlation coefficient.

\section{Results}

Three participants' data were excluded due to an overall accuracy rate below $70 \%$ in experimental blocks. This was an arbitrary limit, set to ensure reasonable adherence to task instructions among included participants. Trials were only included if the previous trial (and also, for the RT analyses, the current trial) was responded to correctly. The first trial of each block was excluded, as were the first trial of each mini-block and the first trial following each mini-block (i.e., trials on which there was a change in preparation interval from the previous trial). Mean RT and percentage errors in each condition are shown in Table 1; switch costs are shown in Figure 2.

RT analysis, 1000 msec preparation interval. The main effect of transition was significant, $F(1,34)=14.32, p=.001, \eta_{p}^{2}=.296$, reflecting an overall cost for switching tasks. Importantly, preceding trial completion did not modify the effect of transition, $F(1,34)$ $=0.08, p=.778, \eta_{p}^{2}=.002$. There was a significant main effect of preceding trial completion, $F(1,34)=182.61, p<.001, \eta_{\mathrm{p}}^{2}=.843$; collapsing across transition, responses 
were $77.0 \mathrm{msec}, 95 \% \mathrm{CI}[65.4,88.6]$, faster following cue-only than following completed trials.

Errors analysis, 1000 msec preparation interval. The overall cost of switching tasks was reflected in a significant main effect of transition, $F(1,34)=9.09, p=.005, \eta_{\mathrm{p}}^{2}=$ .211. Again, preceding trial completion did not modify the transition effect, $F(1,34)=0.07, p$ $=.793, \eta_{\mathrm{p}}^{2}=.002$. The main effect of preceding trial completion was significant, $F(1,34)=$ 23.90, $p<.001, \eta_{\mathrm{p}}^{2}=.413$; collapsing across transition, there were $2.73 \%, 95 \%$ CI [1.59, 3.86], fewer errors made following cue-only than following completed trials

RISC effect analyses, following completed trials only. There was no evidence of a RISC effect (reduction in switch cost at 1000 msec compared with $300 \mathrm{msec}$ preparation interval) following completed trials in these data, either in terms of RT, where the pattern was numerically opposite to a RISC effect, $-7.1 \mathrm{msec}[-16.9,2.7], F(1,34)=2.15, p=.152, \eta_{\mathrm{p}}^{2}=$ .059 , or errors, $0.97 \%[-1.40,3.34], F(1,34)=0.69, p=.410, \eta_{\mathrm{p}}^{2}=.020$.

Correlational analysis (RT). There was no evidence of a correlation between the size of participants' RISC effect scores (following completed trials) and their preparationdriven switch cost scores, $r(33)=.221, p=.203$.

Summary. On trials with a long preparation interval (1000 msec), there was a significant RT cost of switching tasks following cue-only trials which was as large as that following completed trials. In terms of errors, a significant overall switch cost was not modified by preceding trial completion, although the error switch cost following cue-only trials did not itself reach significance (see Figure 2). There was no RISC effect and no evidence of a correlation between RISC and preparation-driven switch costs. Performance 
following cue-only trials was substantially faster and more accurate than that following completed trials.

\section{Discussion}

There was a significant cost of switching tasks subsequent to trials on which only a task-cue was shown and which therefore allowed task-preparation, but not task-based judgement of a target stimulus, response-selection or response-execution to take place. Hence, these results replicate those of Lenartowicz et al. (2011) and demonstrate the effect using different tasks. Previous research indicating that a task had to be used to process a target stimulus for the purposes of selecting a task-appropriate response in order for a subsequent switch cost to be present (Schuch \& Koch, 2003; also Astle et al., 2006, and Los \& Van der Burg, 2010) used the "no-go" method that, as identified by Lenartowicz et al., may render the effects of task-preparation unobservable in terms of subsequent switch-costs. It appears to be the case on the basis of data acquired using the "truncated-trial" method (this study and Lenatrowicz et al., 2011; see also Brass \& von Cramon, 2004 and Desmet et al., 2012 for similar designs) that simply preparing to do a task on the basis of a preparatory cue is sufficient to produce a subsequent switch cost.

We attempted to eliminate a potential confound of cue-switching with task-switching by using two cues for each task. Thus, the switch costs we obtained cannot be due directly to a benefit on repeat trials from repeating a primed cue-word because our repeat trials involved a switch of cue-word from the preceding trial. Nevertheless, using two cues per task might not eliminate all sources of switch effect which are related to processing cues rather than tasks per se. Thus, Logan \& Schneider (2006) showed that even with two cues per task, priming of cue-encoding can take place when cues are semantically related, as was the case 
here. In fact, those authors argued that even entirely unrelated, arbitrary cues can prime each other via a "mediator" concept, such that it might not be possible entirely to separate cuerelated from task-related switch effects in the standard task-switching paradigm.

There was no evidence of a RISC effect in these data - i.e., there was no decrease in switch cost associated with increasing preparation time on the current trial - and also no evidence for a correlation between the size of the RISC effect (following completed trials) and the switch cost driven by preparation on the previous trial. However, it is likely that we were unable properly to compare performance at the two preparation intervals in this experiment because 300 and $1000 \mathrm{msec}$ preparation intervals were presented in runs ("miniblocks") of same-interval trials within experimental blocks. Participants might have made an extra effort to prepare quickly on the $300 \mathrm{msec}$ preparation trials, knowing that they would only have to do so for a few trials in a row, and made relatively less effort on the $1000 \mathrm{msec}$ preparation trials; such differences in strategy would tend to confound our RISC measure. In Experiment 2, we addressed this issue by randomly distributing $300 \mathrm{msec}$ preparation trials throughout the experimental blocks.

Finally, responses following cue-only trials were in general both faster and more accurate than those following completed trials (main effect of preceding trial completion; see Table 1). The same pattern was seen in Lenartowicz et al. (2011, Experiment 2) who suggested that the increased pace of trial events following cue-only trials might have led to a speeding of responses following those trials, with a consequent reduction in attention following completed trials resulting in lower accuracy following those trials. An alternative possibility $^{3}$ is that because there were never two consecutive cue-only trials, there was effectively a decrease in uncertainty concerning the next event following the cue on trials

\footnotetext{
${ }^{3}$ We thank reviewer Sander Los for this suggestion
} 
following cue-only trials (relative to trials following completed trials), which may have led to improved performance. Thus, following a completed trial the current trial might turn out to be either another completed trial or cue-only trial, and therefore the next event following its cue might be either a target stimulus or the another cue. In contrast, following a cue-only trial the current trial could only ever be a completed trial, enabling participants to prepare upon cue presentation with high certainty for the next event being a target, potentially leading to both faster and more accurate responses. We return to this issue in the next experiment.

\section{Experiment 2}

In Experiment 2, we made two changes to the design with the aim of improving its sensitivity. First, we inter-mixed equal numbers of completed trials with each of the two preparation intervals randomly within blocks, rather than grouping them in mini-blocks. Second, we removed the time-limit for responses.

\section{Methods}

Participants. Thirty-seven participants were tested (27 female), either in return for course credit or as unpaid volunteers. The age range was 18-30 years (median 21 years). The experiment was passed by the Ethics Committee of the School of Psychology, University of Aberdeen, and complied with APA ethical standards.

Materials. These were as for Experiment 1 except for the proportion of completed and cue-only trials. As in Experiment 1, among the trials with a 1000-msec preparation interval the proportion of cue-only : completed trials was $30: 70$. But in this experiment we increased the number of trials with a 300-msec preparation interval (all of which were 
completed $^{4}$ ), to match the number of long-preparation completed trials, for a better assessment of the RISC effect. Thus, the final ratio of trial-types (all randomly intermixed within blocks, with the restriction that cue-only trials could not occur consecutively) was 30 :

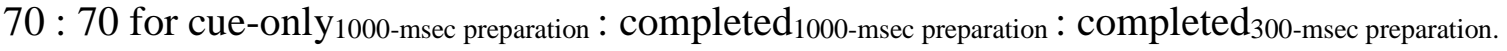

Procedure. This was similar to Experiment 1 but with two key differences. First, trials with a $300 \mathrm{msec}$ preparation interval were no longer presented in mini-blocks but intermixed randomly with the other trials. This meant that participants could not reliably predict the preparation interval of the current trial. Second, there was no longer a time-limit for responding. Instead, after the target was displayed (for $200 \mathrm{msec}$ ), a blank screen was shown until a response was executed. Following the response, a response-stimulus interval occurred of either 600 or $1300 \mathrm{msec}$, the length of which was calculated to balance the cuetarget interval of the subsequent trial (1000 or $300 \mathrm{msec}$, respectively) such that the responsetarget interval on each trial totalled $1600 \mathrm{msec}$. This change meant that trials were no longer of a fixed length.

There were two other minor changes. Practice trial blocks were now all 20 trials long: first, each task was practised in separate blocks (all trials being "completed" trials with a $1000 \mathrm{msec}$ preparation interval), then both tasks were intermixed in a block, and a final block introduced both cue-only trials (with a $1000 \mathrm{msec}$ preparation interval) and $300 \mathrm{msec}$ preparation interval (completed) trials, to mirror the experimental blocks. In addition, experimental blocks (of which there were 32, as previously) were now 56 trials in length; this was to ensure a sufficient number of trials per condition in the changed design.

\footnotetext{
${ }^{4}$ The reason for not including any cue-only trials with a $300 \mathrm{msec}$ preparation interval was that upon piloting it was clear that the cue could not be interpreted in such a short time and the subsequent trial became too difficult. In practice it seemed reasonable not to include such a condition because we were only interested in the subsequent effects of cue-only trials on which there had been sufficient time to prepare the cued task.
} 
Design \& Analysis. The design was entirely within-subjects, analysed by repeatedmeasures ANOVA. The independent variables were preparation interval (300 msec, 1000 msec), preceding trial completion (completed, cue-only) and transition (repeat, switch). The dependent variables were mean response time (RT) on correct trials and mean percentage errors (i.e., percentage of trials within a cell which were responded to with the wrong response), both measured only on completed trials. All statistical tests were 2-tailed, with an $\alpha$-level of 0.05 .

Mean RTs and percentage errors (from completed trials only) were calculated within each condition for each participant; the means of these values across participants are shown in Table 1. Mean switch costs (with 95\% confidence interval, calculated as in Experiment 1) are shown in Figure 2.

Again, we correlated individual participants' RISC effect scores, measured on trials following completed trials, with their preparation-driven switch cost scores, measured following cue-only trials on trials with both $300 \mathrm{msec}$ and $1000 \mathrm{msec}$ preparation intervals (in separate analyses), using the Pearson product-moment correlation coefficient. N.B. We do not report the analogous analysis using the RISC effect following cue-only trials since the same data would contribute to the values on both sides of such an analysis and therefore any significant correlation would be likely to be meaningless.

\section{Results}

The data from five participants were excluded due to an overall accuracy rate of less than $70 \%$ in experimental blocks; the remaining 32 datasets were analysed as detailed above. The first trial of each block was excluded, as were trials following an error and (for the RT 
analyses only) trials on which an error was made. In order to avoid a confound between the two preceding trial completion conditions in terms of the preceding trial's preparation interval (because completed trials could use either interval whereas cue-only trials only ever used the 1000 msec interval), we only analysed trials on which the preceding preparation interval was $1000 \mathrm{msec}$. Trials with RTs $2000 \mathrm{msec}$ or above were trimmed from the dataset, since very slow trials may represent a type of error and are likely to cause skewing of statistical analyses, and we also excluded trials which immediately followed such trials. Lenartowicz et al. (2011) also trimmed data using a 2000 msec RT limit. The mean percentage of trimmed trials (RT > $2000 \mathrm{msec}$ ) per participant was $5.8 \%$, range $0.0 \%-$ 25.1\%. It is clear that some participants had a high percentage of trials trimmed. Therefore, to ensure that our analyses were not unduly affected by a small number of unrepresentative participants, we re-ran all analyses after excluding the five participants with more than $10 \%$ trials trimmed (mean $3.3 \%$ trials trimmed, range $0.0 \%-9.4 \%$, post-exclusions), as well as with an alternative trimming threshold of $5000 \mathrm{msec}$ (all participants included; mean $0.6 \%$ trials trimmed, range $0.0-6.9 \%$ ). The pattern of results was very similar across all three methods.

Mean RT and percentage errors in each condition are shown in Table 1; switch costs are shown in Figure 2.

RT. Overall, the cost of switching tasks was highly significant, $F(1,31)=27.41, p<$ $.001, \eta_{\mathrm{p}}^{2}=.469$, and not significantly modified by preceding trial completion, $F(1,31)=1.97$, $p=.170, \eta_{\mathrm{p}}^{2}=.060$. The main effect of preceding trial completion was significant, $F(1,31)=$ $31.42, p<.001, \eta_{p}^{2}=.503$, with faster responses following cue-only than following completed trials as in Experiment 1. The main effect of preparation interval was significant, $F(1,31)=$ $70.57, p<.001, \eta_{\mathrm{p}}^{2}=.695$, with faster responses at the $1000 \mathrm{msec}$ than the $300 \mathrm{msec}$ 
preparation interval. A significant interaction between transition and preparation interval, $F(1,31)=6.22, p=.018, \eta_{\mathrm{p}}^{2}=.167$, was due to the presence of an overall RISC effect, but this effect was significantly modified by preceding trial completion, as shown by a significant 3 -way interaction, $F(1,31)=8.92, p=.005, \eta_{p}^{2}=.224$. Examination of this interaction revealed that there was no RISC effect following completed trials, $-4.4 \mathrm{msec}, 95 \%$ CI [-31.7, 22.8], $F(1,31)=0.11, p=.742, \eta_{\mathrm{p}}^{2}=.004$, but a significant RISC effect following cue-only trials, $48.5 \mathrm{msec}[24.9,72.1], F(1,31)=17.56, p<.001, \eta_{\mathrm{p}}^{2}=.362$. The switch cost at the 300 msec preparation interval was significantly larger following cue-only trials than following completed trials, a difference of $37.4 \mathrm{msec}[12.6,62.2], F(1,31)=9.47, p=.004$, $\eta_{\mathrm{p}}^{2}=.234$; at the $1000 \mathrm{msec}$ preparation interval the corresponding difference was $-15.5 \mathrm{msec}$ $[-38.8,7.9], F(1,31)=1.83, p=.186, \eta_{\mathrm{p}}^{2}=.056$. There was a significant 2-way interaction between preparation interval and preceding trial completion, $F(1,31)=42.99, p<.001$, $\eta_{\mathrm{p}}^{2}=$ .581 , and the significant 3-way interaction, noted above, showed that this pattern was also affected by the transition factor. The interaction between preparation interval and preceding trial completion was significant both on repeat trials, $F(1,31)=15.60, p<.001, \eta_{\mathrm{p}}^{2}=.335$, and switch trials, $F(1,31)=43.16, p<.001, \eta_{\mathrm{p}}^{2}=.582$. On repeat trials there was a significant benefit for the preceding trial being cue-only as opposed to completed at the long preparation interval, a difference of $79.4 \mathrm{msec}[56.5,102.4]$; this benefit was smaller but still significant at the short interval, a difference of $24.0 \mathrm{msec}[0.3,47.7]$. On switch trials there was a significant benefit for the preceding trial being cue-only only at the $1000 \mathrm{msec}$ interval, a difference of $94.9 \mathrm{msec}[69.9,120.0]$; at the shorter interval the difference of $-13.4 \mathrm{msec}$ [$38.8,12.0]$ was not significant. 
Errors. Overall, the cost of switching tasks was significant, $F(1,31)=21.85, p<$ $.001, \eta_{\mathrm{p}}^{2}=.413$. Whilst the switch cost was not significantly modified by preceding trial completion, there was a non-significant tendency towards smaller switch costs following cueonly trials than following completed trials, $F(1,31)=3.51, p=.070, \eta_{p}^{2}=.102$. The main effect of preceding trial completion was not significant, $F(1,31)=2.28, p=.141, \eta_{p}^{2}=.069$. The main effect of preparation interval was not significant, $F(1,31)=1.23, p=.277, \eta_{\mathrm{p}}^{2}=$ .038 , and neither did preparation interval interact with transition, $F(1,31)<0.01, p=.964, \eta_{\mathrm{p}}^{2}$ $<.001$; thus, no RISC effect was present in the error data. A significant interaction between preparation interval and previous trial completion, $F(1,31)=11.47, p=.002, \eta_{\mathrm{p}}^{2}=.270$, was due to there being fewer errors following cue-only than following completed trials at the 1000 msec preparation interval, 2.16\%, 95\% CI [0.96, 3.35], but no benefit (in fact a slight numerical cost) at the $300 \mathrm{msec}$ preparation interval, $-0.38 \%[-2.00,1.25]$. The three-way interaction was not significant, $F(1,31)=0.13, p=.726, \eta_{\mathrm{p}}^{2}=.004$.

Correlational analysis (RT). There was no evidence of a correlation between the size of participants' RISC effect scores (following completed trials) and their preparationdriven switch cost scores either on trials with a $300 \mathrm{msec}$ preparation interval, $r(30)=.052, p$ $=.777$, or with a 1000 msec preparation interval, $r(30)=.062, p=.734$.

Summary. As in Experiment 1, significant switch costs were present following both completed and cue-only trials. At the short preparation interval, the switch cost following cue-only trials was significantly larger than that following completed trials, and there was a significant RISC effect only following cue-only trials. There was no evidence of a correlation between RISC effect (following completed trials) and size of switch cost following cue-only trials. In terms of both the RT and error data, there was a benefit to 
performance associated with the preceding trial being a cue-only trial rather than a completed trial; this benefit was only present for long-preparation trials.

\section{Discussion}

In Experiment 1 we had been unable to demonstrate a significant RISC effect alongside a preparation-driven switch cost (i.e., one seen following cue-only trials); this seemed odd given that one would expect that a task-preparation process strong enough to generate a switch cost on a subsequent trial would also be strong enough to reduce the same cost on the current trial. In Experiment 2, however, there was both a substantial switch cost following cue-only trials and a substantial reduction of that same cost with a long (1000 msec) preparation interval on the current trial. This pattern of results replicates that seen in Lenartowicz et al. (2011).

We found no RISC effect at all following completed trials. Whilst we had not predicted this result, we note that same was true (at least in terms of statistical significance) in the study of Lenartowicz et al. (2011). In fact, when we look at the values from that study from the trial-types most comparable to those analysed in our own study (i.e., trials preceded by a long preparation interval), we see a similar picture to that presented here. Thus, in the Lenartowicz et al. study, switch costs following cue-only trials were $113 \mathrm{msec}$ at the shorter (350 $\mathrm{msec})$ preparation interval, falling steeply to $25 \mathrm{msec}$ at the longer $(1250 \mathrm{msec})$ preparation interval, whereas following completed trials (their "go" trials) switch costs were just $63 \mathrm{msec}$ at the shorter preparation interval with a small numerical reduction to $53 \mathrm{msec}$ at the longer preparation interval. 
Lenartowicz et al. suggested that the faster pace of events following cue-only trials may have meant a relative reduction in attention on standard go trails, leading to a reduced RISC effect following go (completed) trials (as well as poorer performance overall). Whilst such a mechanism may have abolished the RISC effect following completed trials in our study also, it does not seem to fully capture the pattern that appears to be present in both experiments. That is, it is not simply that there was a relative failure to reduce switch costs when a long preparation time was available in which to do so, but rather (or in addition) that in both studies switch costs at the short preparation interval were actually considerably larger (roughly twice the size) following cue-only trials than following go/completed trials. In fact, the absence of the RISC effect following completed trials might be due primarily to the small size of the switch cost at the shorter interval, such that there was little room for it to be reduced with a longer interval, rather than to a lack of reduction per se.

The idea that switch costs might be larger following preparation than following performance on the preceding trial is striking, as it is the opposite of what we have come to expect following studies using no-go methodology. There are some aspects of the design which we and Lenartowicz et al. used which may have contributed to this effect. First (c.f. Lenartowicz et al.), the cue-cue interval was always shorter on cue-only/completed trial-pairs than completed/completed trial-pairs, simply because cue-only trials were themselves much shorter. If task-activation declines passively following the cueing event (e.g. Altmann, 2002) it would have less time to decline by the end of a cue-only trial than it would by the end of a completed trial, and this might lead to a greater difficulty in switching away from that task following a cue-only trial than following a completed trial. Second, a response-cue interval (at least $600 \mathrm{msec}$ in both studies) occurred only after the end of completed trials, during which participants might have passively (Meiran, Chorev \& Sapir, 2000) or actively (Monsell, Sumner, \& Waters, 2003) disengaged from the just-used task prior to the next trial, 
reducing subsequent switch costs. Third, participants may have been more ready to engage in task-preparation following completed than cue-only trials, and therefore able to prepare more rapidly or effectively during the short preparation interval on the subsequent trial. Such a difference in readiness could have come about because the end of completed trials was clearly signalled by the response, and it was followed (after the response-cue interval) by a subsequent cue with $100 \%$ likelihood; participants might therefore have been relatively quite ready to begin cue-processing and, if necessary, task-switching, as soon as the cue appeared following the response on a completed trial. In contrast, cue-only trials consisted only of a preparation period, identical to that in the more common completed trials; at the end of cueonly trials, therefore, participants would tend to expect a target, rather than another cue, to be presented next, and might be relatively unprepared to process a subsequent cue, or to use it to switch tasks, within the $300 \mathrm{msec}$ allowed on short-preparation trials.

Again, we found no evidence for a correlation between RISC scores (following completed trials) and switch costs following cue-only trials across individual participants. This lack of correlation may indicate that the same preparatory process does not in fact underlie both the generation of switch costs subsequent to cue-only trials and the reduction of existing switch costs following completed trials. However, as stated above, we did not capture any overall RISC effect following completed trials in our design, and it may be that RISC and preparation-driven switch costs can be shown to correlate using a different design which is able to generate a substantial RISC effect following completed trials.

The RT switch costs at the 1000 msec preparation interval reached significance following both cue-only and completed trials and might therefore be considered to be "residual" in nature. However, as noted in Swainson \& Martin (2013), it is difficult to claim strongly that switch costs are residual unless they have been shown to remain stable across more than one preparation interval, because in principle an even longer preparation might 
have reduced the switch cost further. To look ahead, there was stronger evidence of residual costs in Experiment 3, and so this issue will be returned to below.

As in Experiment 1, we saw again that there was a benefit to overall performance (i.e., affecting both switch and repeat trials) associated with the preceding trial having been a cue-only trial as opposed to a completed trial, but it became clear in the current experiment that this benefit only applied when the (current) trial had a 1000 msec preparation interval. (N.B. As this interaction does not involve the transition effect, it cannot be seen in Figure 2; see Results section for relevant data values, collapsed across transition). The restriction of the benefit to trials with $1000 \mathrm{msec}$ preparation time suggests it took time to take effect following the preceding cue-only trial, as might be expected for both of the possibilities suggested above: i.e., the recruitment of attention stemming from a faster pace of events (c.f. Lenartowicz et al., 2011), and the effective preparation for an imminent target following the elimination of uncertainty that the next event would in fact be a target. We will return again to this issue in Experiment 3.

Whilst the data presented so far indicate that having the opportunity to prepare a task even in the absence of a target stimulus is sufficient to trigger a substantial cost of switching tasks on the subsequent trial, they do not indicate which aspect(s) of task-preparation may be necessary to trigger the subsequent switch cost. We tackled this question in Experiment 3. 


\section{Experiment 3}

The pattern of a reduction in switch cost with increasing preparation time (RISC effect) which we saw following cue-only trials in Experiment 2 is often described as resulting from a process of "task-set reconfiguration" (Rogers \& Monsell, 1995), usually considered to involve activation of the prepared task's stimulus-mapping rules (e.g., for the colour task: blue/yellow-left; red/green-right). In Experiment 3, we examined whether switch costs would still be observed following cue-only trials if task-cues could not be used to prepare any relevant stimulus-response mappings. We made use of a previous design (Swainson \& Martin, 2013) in which task-cues indicated only which visual dimension (i.e., colour or shape) of a target stimulus ought to be attended to, and the response simply had to be compatible (left-left, right-right) with the location of the task-relevant target feature (e.g. circle) shown on a post-target response-mapping screen. There was no consistent relationship between any particular target feature and any overt manual response across trials, and no stimulus-response mappings associated with the tasks. We predicted that the large, reducible part of the RT switch cost seen following cue-only trials in Experiment 2 would be absent in this experiment, along with any RISC effect, following both completed and cueonly trials. If the residual cost is the result of task-performance on the preceding trial (e.g. response-selection, Schuch \& Koch, 2003, or target-judgement, Swainson \& Martin, 2013), then switch costs at the long preparation interval should be present following completed trials but not following cue-only trials.

\section{Methods}

Participants. Thirty-nine participants were tested (29 female), either in return for course credit or as unpaid volunteers. The age range was 17-34 years (median 20 years). 
The experiment was passed by the Ethics Committee of the School of Psychology, University of Aberdeen, and complied with APA ethical standards.

Materials. The major difference from Experiment 2 was the inclusion of a "response-mapping screen" (Swainson \& Martin, 2013) to allow us to eliminate the stimulusresponse mapping component from the task definitions. The cues (e.g., "COLOUR”) and target stimuli (e.g., a red circle) were identical to those in Experiments 1 and 2. Participants were required to use the cue to identify either the colour or the shape of the upcoming target stimulus. Following presentation of the target, the response-mapping screen was presented, showing a white shape and coloured bar on the left side of the screen and a different shape and colour on the right side (see Figure 1). Participants needed to locate the identified feature and make the spatially compatible response (e.g., following a COLOUR cue and a red triangle target, if the left bar was red, they would press the button under their left index finger). The two features of the target stimulus were both among the items shown on the response-mapping screen, together with an alternative shape and an alternative colour. The combination of each particular colour and shape to the left and right of the screen was random on each trial.

Procedure. Trial event timings were as for Experiment 2, except that instead of a blank screen being shown after the target until a response was made, the response-mapping screen was shown instead (see Figure 1). All other session details were as for Experiment 2.

Design \& Analysis. These were identical to Experiment 2.

\section{Results}

No datasets were excluded on the basis of overall accuracy as all participants achieved an accuracy level of at least $70 \%$ in experimental blocks. The first trial of each 
block was excluded, as were trials following an error and (for the RT analyses only) trials on which an error was made. As in Experiment 2, we only analysed trials on which the preceding preparation interval was $1000 \mathrm{msec}$. Trials with RTs $2000 \mathrm{msec}$ or above were trimmed from the dataset; the mean percentage of trials trimmed per participant was $1.0 \%$, range $0.0 \%-7.7 \%$. Finally, we also required that the RT of the preceding trial, if completed, was below 2000 msec.

Mean RT and percentage errors in each condition are shown in Table 1; switch costs are shown in Figure 2.

RT. There was a significant overall cost of switching tasks, $F(1,38)=33.45, p<$ $.001, \eta_{\mathrm{p}}^{2}=.468$, which was not modified by preceding trial completion, $F(1,38)=2.16, p=$ $.150, \eta_{\mathrm{p}}^{2}<.054$. The main effect of preparation interval was significant, $F(1,38)=82.39, p<$ $.001, \eta_{\mathrm{p}}^{2}=.684$, with faster responses at the longer preparation interval, but there was no evidence of a RISC effect in these data, $F(1,38)<.001, p=.970, \eta_{p}^{2}<.001$. The main effect of preceding trial completion was significant, $F(1,38)=5.62, p=.023, \eta_{\mathrm{p}}^{2}=.129$, with slower responses overall following completed than following cue-only trials, but as in Experiment 2, there was a significant interaction of preceding trial completion with preparation interval, $F(1,38)=23.50, p<.001, \eta_{p}^{2}=.382:$ at the $1000 \mathrm{msec}$ preparation interval, there was again a benefit for the preceding trial having been cue-only rather than completed, a difference of $34.1 \mathrm{msec}, 95 \%$ CI [20.5, 47.8], which was not present at the $300 \mathrm{msec}$ preparation interval where there was a difference of $-5.1 \mathrm{msec}[-21.0,10.9]$; this replicates the pattern seen in Experiment 2. The 3-way interaction was not significant, $F(1,38)=2.58, p=.117 \eta_{\mathrm{p}}^{2}=.064$. 
Errors. The overall cost of switching tasks was significant, $F(1,38)=40.72, p<$ $.001, \eta_{\mathrm{p}}^{2}=.517$, and was not modified by preceding trial completion, $F(1,38)=2.12, p=.153$, $\eta_{\mathrm{p}}^{2}=.053$. There was no main effect of preparation interval, $F(1,38)=0.48, p=.492, \eta_{\mathrm{p}}^{2}=$ .013 , and no interaction of preparation interval with transition, $F(1,38)=0.43, p=.517, \eta_{p}^{2}=$ .011 , therefore no RISC effect. The main effect of preceding trial completion was not significant, $F(1,38)=0.24, p=.627, \eta_{\mathrm{p}}^{2}=.006$. There was, however, a significant interaction between preparation interval and preceding trial completion, $F(1,38)=23.46, p<.001, \eta_{\mathrm{p}}^{2}=$ .382: at the $1000 \mathrm{msec}$ preparation interval, there was a significant benefit to the preceding trial being cue-only as opposed to completed, a difference of $1.64 \%, 95 \%$ CI $[0.04,3.25]$; at the 300 msec preparation interval, in contrast, the effect was reversed with a significant cost to the preceding trial being cue-only as opposed to completed, a difference of $-2.28 \%[-3.79$, -0.77]. The 3-way interaction was not significant, $F(1,38)=1.53, p=.224, \eta_{\mathrm{p}}^{2}=.039$.

Correlational analysis (RT). There was no evidence of a correlation between the size of participants' RISC effect scores (following completed trials) and their preparationdriven switch cost scores either on trials with a $300 \mathrm{msec}$ preparation interval, $r(37)=.044, p$ $=.789$, or with a 1000 msec preparation interval, $r(37)=-.201, p=.221$.

Between-experiments analysis of "reducible switch cost". Whereas in Experiment 2 we had found a significant reducible RT switch cost following cue-only trials, there was none apparent in the analysis of Experiment 3 data described above. In order formally to determine the size of this measure in each experiment, and to establish whether it differed across the two experiments, we defined the "reducible switch cost" as the difference in magnitude of the switch cost between the $300 \mathrm{msec}$ and $1000 \mathrm{msec}$ preparation intervals. In Experiment 2 the reducible switch cost following cue-only trials was $48.5 \mathrm{msec}, 95 \% \mathrm{CI}$ 
$[24.9,72.1]$ and in Experiment 3 it was 8.8 msec $[-10.4,28.0]$; the difference between experiments of $39.7 \mathrm{msec}[10.2,69.2]$ was significant, $t(69)=2.681, \mathrm{p}=.009, \eta_{\mathrm{p}}^{2}=.094$.

Summary. Switch costs were statistically significant following cue-only trials in both RT and error scores. There was no RISC effect, so switch costs following both types of task-completion trial can be considered likely to be residual. The absence of a reducible switch cost following cue-only trials contrasted with its presence in Experiment 2. There was no evidence of a correlation between RISC effect (following completed trials) and size of switch cost following cue-only trials. The preceding trial being a cue-only trial conferred a benefit to performance on trials with a long preparation interval, but a cost (significant in terms of errors only) on trials with a short preparation interval.

\section{Discussion}

We had hypothesised that the large, reducible switch costs seen following cue-only trials in Experiment 2 had resulted from preparation of the prepared tasks' stimulus-response mappings and that they should therefore be absent if no stimulus-response mappings could be prepared during the preparation interval. In line with that prediction, we found no evidence for a reducible switch cost in this experiment. The difference between experiments on this measure was presumably due to the fact that the task-definitions in Experiment 2 included specific stimulus-response mappings whereas those in Experiment 3 did not; nevertheless, we cannot yet definitively conclude that there was no reducible switch cost at all in Experiment 3 because it may be that we did not use a short enough preparation interval to be able to observe it: as discussed above, it is possible that a reducible switch cost is able to be 
overcome during the $300 \mathrm{msec}$ preparation interval used in these experiments. Overcoming a reducible switch cost could potentially be especially rapid in Experiment 3 because the taskdefinitions involved no specific stimulus-response mappings. The issue of whether any reducible switch cost at all could potentially be generated by either the preparation or performance of tasks lacking stimulus-response mappings must therefore be resolved via future experiments. The absence of a RISC effect following completed trials in this experiment, as in Experiments 1 and 2, may also explain the lack of a significant correlation between RISC effect (following completed trials) and size of switch costs following cue-only trials.

The switch costs present in this experiment appeared to be residual in nature in that they were not significantly smaller at the $1000 \mathrm{msec}$ preparation interval than at the $300 \mathrm{msec}$ interval. We had not predicted that residual switch costs would be present following cueonly trials in any of these experiments. By definition, the residual switch cost can only be overcome by performing the new task, and not simply by preparing for it; i.e., it provides evidence that some part of the switching process cannot completed by volition alone (Rogers \& Monsell, 1995). Its presence has been ascribed to the exogenously-driven switch of alternative task-rules within procedural working memory (e.g., Rubinstein, Meyer, \& Evans, 2001). Therefore it would seem to make sense to expect that if any part of the total switch cost would require the performance, and not solely the preparation, of a task on the preceding trial, it would be the residual switch cost. The studies of Schuch \& Koch (2003) and Los \& Van der Burg (2010), both showing residual costs only after task-performance but not after a no-go response, provide supporting evidence for that view. The demonstration by Swainson \& Martin (2013) that the selection of an overt response is not necessary to trigger a subsequent, probably residual, switch cost - costs being present at long preparation intervals following covert responses (mental selection of a task-based stimulus feature) - still allows 
for $u$ sing the task being critical to establishing the conditions for a subsequent residual switch cost (see also Los \& Van der Burg, 2010). In contrast, the current data suggest the possibility that the residual cost (or a part of it) requires neither overt nor covert performance of a task, nor the preparation of a task's stimulus-response mappings, but that it can be driven by some other process involved with, or initiated by, processing a task-cue. Hence, we must look for other explanations for the presence of this cost.

One possible explanation for the presence of residual switch costs following cue-only trials is that they represent a failure to cancel the activation of the task which was cued but not performed on the preceding trial. Evidence that unfulfilled intentions that are explicitly no longer relevant can impact upon future performance lends support to this idea (Bugg \& Scullin, 2013). For instance, it may be that task-preparation sets up an intention in prospective memory for the prepared task to be applied to the next target that is presented (which, following a cue-only trial, would be the subsequent trial's target) and that this intention is not automatically cancelled when an alternative intention (driven by the cue on that subsequent trial) is set up. If so, two contradictory task-sets could be triggered by the target on a switch trial following a cue-only trial, resulting in a delay whilst a single task-set is selected to control response-selection, and leading to an RT switch cost. This situation could potentially pertain to all switch trials following cue-only trials, with an obligatory delay being caused by the need to resolve task-selection; alternatively, just as successful preparation on a switch trial may be probabilistic (de Jong, 2000), so the cancellation of the preceding trial's task may also be probabilistic, with the residual switch cost following cueonly trials resulting from a mixture of trials where the preceding trial had been cancelled and trials where it had not.

An alternative explanation for the presence of residual switch costs following cue-only trials is that the residual RT switch costs observed in this experiment (and possibly more 
widely in the task-switching literature) might result from a strategic slowing of responding to cope with the relative uncertainty of having two different tasks cued within a relatively short space of time. This idea resembles the effect of the "change-detector" element in the computational model of Brown, Reynolds and Braver (2007), proposed to initiate a slowing of responses on switch trials (and successive trials, be they switch or repeat) that contributed to the residual switch cost. While Brown et al.'s model was designed to apply to the situation of switching from one performed task to another, the same mechanism might potentially also apply to a switch from a task which was prepared but not executed.

As in Experiment 2, in the current experiment we saw an interaction between preparation interval and preceding trial completion. (See Results section for relevant data values, collapsed across transition). At the long preparation interval, there was a benefit to performance from the preceding trial being a cue-only as opposed to a completed trial, both in terms of RT and errors, just as there had been in Experiments $1 \& 2$. At the short preparation interval, there was no benefit; in fact, in terms of errors there was a significant cost of the preceding trial being cue-only. Where might this cost stem from? One possibility is that when the preceding trial had a long preparation interval and the current trial a short preparation interval, the shift in preparation time was in some way disruptive, leading to an increase in errors. Trials following cue-only trials might be particularly susceptible to such disruption because the two cueing intervals follow on immediately one from the other following cue-only trials, whereas following completed trials the target, response-mapping screen and response events all intervene between the two cueing intervals. N.B. The interactions between preparation interval and preceding trial completion were not the focus of these studies, and they had not been predicted; our explanations for them are therefore speculative at this stage. 


\section{General Discussion}

In these studies, we asked what it is that needs to happen in terms of task-processing on one trial in order to establish the conditions for a switch cost to be present on the next trial. In all three experiments, switch costs were present following cue-only trials; thus, it is clear that task-preparation is sufficient to drive subsequent switch costs, with no need for the processing of a target stimulus or the selection of a task-appropriate response to have taken place. Furthermore, task-preparation appeared to be sufficient to trigger both that part of the subsequent switch cost which is reducible with preparation on the current trial and the residual part of the switch cost which remains despite substantial preparation. Notably, a residual switch cost was present following trials on which not only was no task performed but also no task-specific stimulus-response mappings prepared.

The main point to be drawn from these results is that remarkably little task-processing seems to be necessary to trigger a subsequent switch cost. While Schuch and Koch (2003; see also Los \& Van der Burg, 2010) posited that applying a task to a target stimulus in order to select an appropriate response was necessary to trigger a subsequent switch cost, our data indicate not only that simply preparing to perform one task rather than another may be sufficient (replicating Lenartowicz et al., 2011), but also that preparation need not even involve the specification of stimulus-response mappings.

As Lenartowicz et al. (2011) pointed out, it would be rather paradoxical if we were able to prepare a cued task in advance of a target in such a way that the switch cost on the current trial was at least partially overcome (giving the RISC effect) but if that same preparation led to no switch cost at all on a subsequent trial, although this is what has appeared to be the case in a number of studies using no-go stimuli (Astle et al., 2006; Schuch 
\& Koch, 2003; Los \& Van der Burg, 2010). Lenartowicz et al. showed that in fact a large switch cost could be seen subsequent to a cue-only trial (i.e., a trial that was truncated immediately after the cue-target interval, without any no-go stimulus being presented), and we replicated that finding here whilst avoiding a potential confound from direct cuerepetitions on task-repeat trials (although see below regarding a related cue-priming confound which might remain; see also Brass \& von Cramon, 2004 and Desmet et al., 2012 for similar findings with a "double-cue" design). This large preparation-driven switch cost, reducible with subsequent preparation, was present in Experiment 2 but not in Experiment 3. Since only in Experiment 2 were tasks defined in terms of stimulus-response mappings, this pattern of results is consistent with the reducible part of the switch cost being related to the need to reconfigure stimulus-response mappings prior to task-execution. It seems clear, given the results of these and previous experiments that the reducible part of the switch cost can be driven by task-preparation alone, and does not require that any task-performance took place on the preceding trial.

Perhaps more surprising is our finding that the residual switch cost appeared to be present in our data following cue-only trials. By definition, the residual switch cost can only be overcome by performance of a task, and according to Schuch \& Koch (2003) it is present because of task-performance (specifically, response-selection) on the preceding trial. Our results suggest the possibility that this cost (or at least a measurable part of it) need not be driven by task-performance, nor even by preparation of a task's stimulus-response mappings. It seems initially rather counter-intuitive that forming an intention to perform according to a particular task should produce an effect which is not subsequently easily overturned with (presumably) sufficient time and information to enable the formation of a contradictory intention. We have speculated on two possible reasons why this cost might occur: first, it may be that intentions are not as easily abolished as we might expect (c.f. Bugg \& Scullin, 
2013), such that following a cue-only trial two intentions may be effective at the time of target-presentation; second (and possibly as a consequence of the persistence of unfulfilled intentions), switching from one intention to another following a cue-only trial may trigger a shift to a more cautious mode of responding analogous to that triggered by switching from one performed task to another (c.f. Brown et al., 2007).

There was no evidence in the data reported here for any additional contribution to the size of switch costs being made by task-processing stages subsequent to preparation: i.e., in none of the three experiments presented here did we find that switch costs following completed trials were significantly larger than those following cue-only trials. Such an additional contribution might have been expected to affect particularly residual switch costs; however, it may be that the relatively small size of the residual switch costs in these experiments simply did not leave much opportunity for differences in their sizes based on the type of preceding trial to become noticeable. Alternatively, it may be that there is actually no contribution to the size of subsequent switch costs made by target-processing, responseselection or response-execution, and that we should put previous reports of such effects (e.g. Schuch \& Koch, 2003; Astle et al., 2006; Los \& Van der Burg, 2010; Philipp et al., 2007) down to the use in those studies of no-go stimuli, which may have prevented the subsequent effects of earlier stages from being seen (Lenartowicz et al., 2011). But whilst we obtained no evidence of post-preparation stages leading to an increased subsequent switch cost, we did observe a significant effect in the opposite direction: that is, we saw significantly larger RT switch costs at the 300-msec preparation interval following cue-only trials than following completed trials (Experiment 2). This same effect appears to have been present (although it was not necessarily statistically significant) in the Lenartowicz et al. study. This is clearly an intriguing finding, suggesting the possibility that, far from switch costs being necessarily absent unless the preceding trial's task was performed, costs might actually be bigger 
following preparation than following performance on the preceding trial. However, as explained above, it remains possible that this effect was caused, or contributed to, by procedural differences between cue-only and completed trials in terms of timing and predictability. This is clearly an issue that should be addressed in future experiments.

To what extent might the switch costs that we saw following cue-only trials result from priming of cue-encoding processes, such as has been suggested to occur between semantically associated and even arbitrary cues (Logan \& Schneider, 2006), as opposed to being "true" task-switch effects? On the basis of our current data, we cannot say. Whilst we eliminated the repetition of identical cue-words across repeat trials, the switch costs that we measured might still be capturing an effect which is more to do with switching between cuerelated information than between tasks. It might be that a design such as that developed by Arrington, Logan \& Schneider (2007), requiring separate responses to cues and targets, could help to determine whether any switch effect definitively indexes "true" task-switching as opposed to cue-priming.

\section{Conclusion}

Truncating trials at the stage of task-preparation led to subsequent switch costs. Preparing specific stimulus-response mappings on cue-only trials led to subsequent RT costs which were larger than those following completed trials when the preparation interval on the current trial was short and which reduced with increasing preparation time. Preparing only to attend to the appropriate dimension of target stimuli led to subsequent, and apparently residual, RT switch costs which we speculate may be due to the persistence of unfulfilled task intentions and/or the adoption of a cautious response-slowing strategy when consecutive cues indicated discrepant tasks. 


\section{References}

Altmann, E. M. (2002). Functional decay of memory for tasks. Psychological Research, 66, 287-297. doi:10.1007/s00426-002-0102-9

Altmann, E. M. (2004). The preparation effect in task switching: Carryover of SOA. Memory and Cognition, 32(1), 153-163.

Arrington, C. M., Logan, G. D., \& Schneider, D. W. (2007). Separating cue encoding from target processing in the explicit task-cuing procedure: Are there "true" task switch effects? Journal of Experimental Psychology.Learning, Memory, and Cognition, 33(3), 484-502. doi:2007-06096-003

Astle, D. E., Jackson, G. M., \& Swainson, R. (2006). Dissociating neural indices of dynamic cognitive control in advance task-set preparation: An ERP study of task switching. Brain Research, 1125, 94-103. doi:10.1016/j.brainres.2006.09.092

Brass, M., \& von Cramon, D. Y. (2004). Decomposing components of task preparation with functional magnetic resonance imaging. Journal of Cognitive Neuroscience, 16(4), 609620. doi:10.1162/089892904323057335

Brown, J. W., Reynolds, J. R., \& Braver, T. S. (2007a). A computational model of fractionated conflict-control mechanisms in task-switching. Cognitive Psychology, 55(1), 37-85. doi:S0010-0285(06)00072-7

Bugg, J. M., \& Scullin, M. K. (2013). Controlling intentions: The surprising ease of stopping after going relative to stopping after never having gone. Psychological Science, 24(12), 2463-2471. doi:10.1177/0956797613494850 
Cedrus Corporation. (2003). RB-730 response pad [computer hardware]. San Pedro, California

Desmet, C., Fias, W., \& Brass, M. (2012). Preparing or executing the wrong task: the influence on switch effects. $Q J$ Exp Psychol, 65, 1172-1184. doi:10.1080/17470218.2011.645840

Kiesel, A., Steinhauser, M., Wendt, M., Falkenstein, M., Jost, K., Philipp, A. M., \& Koch, I. (2010). Control and interference in task switching--a review. Psychological Bulletin, 136(5), 849-874. doi:10.1037/a0019842

Lenartowicz, A., Yeung, N., \& Cohen, J. D. (2011). No-go trials can modulate switch cost by interfering with effects of task preparation. Psychological Research, 75(1), 66-76. doi:10.1007/s00426-010-0286-3

Logan, G. D., \& Bundesen, C. (2003). Clever homunculus: Is there an endogenous act of control in the explicit task-cuing procedure? Journal of Experimental Psychology: Human Perception and Performance, 29(3), 575-599. doi: 10.1037/0096-1523.29.3.575

Logan, G. D., \& Schneider, D. W. (2006). Priming or executive control? Associative priming of cue encoding increases "switch costs" in the explicit task-cuing procedure. Memory \& Cognition, 34(6), 1250-1259. doi: 10.3758/BF03193269

Los, S. A., \& Van der Burg, E. (2010). The origin of switch costs: Task preparation or task application? Quarterly Journal of Experimental Psychology (2006), 63(10), 1895-1915. doi:10.1080/17470211003651849 
Mayr, U., \& Kliegl, R. (2003). Differential effects of cue changes and task changes on taskset selection costs. Journal of Experimental Psychology: Learning Memory and Cognition, 29(3), 362-371. doi: 10.1037/0278-7393.29.3.362

Meiran, N. (1996). Reconfiguration of processing mode prior to task performance. Journal of Experimental Psychology: Learning Memory and Cognition, 22(6), 1423-1442.

Meiran, N., Chorev, Z., \& Sapir, A. (2000). Component processes in task switching. Cognitive Psychology, 41(3), 211-253.

Monsell, S. (2003). Task switching. Trends in Cognitive Sciences, 7(3), 134-140. doi: $10.1016 / \mathrm{S} 1364-6613(03) 00028-7$

Monsell, S., \& Mizon, G. A. (2006). Can the task-cuing paradigm measure an endogenous task-set reconfiguration process? Journal of Experimental Psychology.Human Perception and Performance, 32(3), 493-516. doi:2006-08586-001

Monsell, S., Sumner, P., \& Waters, H. (2003). Task-set reconfiguration with predictable and unpredictable task switches. Memory and Cognition, 31(3), 327-342. doi: 10.3758/BF03194391

Philipp, A. M., Jolicoeur, P., Falkenstein, M., \& Koch, I. (2007). Response selection and response execution in task switching: Evidence from a go-signal paradigm. Journal of Experimental Psychology.Learning, Memory, and Cognition, 33(6), 1062-1075. doi:10.1037/0278-7393.33.6.1062

Rogers, R. D., \& Monsell, S. (1995). Costs of a predictable switch between simple cognitive tasks. Journal of Experimental Psychology: General, 124, 207-231. 
Rubinstein, J. S., Meyer, D. E., \& Evans, J. E. (2001). Executive control of cognitive processes in task switching. Journal of Experimental Psychology: Human Perception and Performance, 27(4), 763-797. doi: 10.1037//0096-1523.27.4.763

Schuch, S., \& Koch, I. (2003). The role of response selection for inhibition of task sets in task shifting. Journal of Experimental Psychology: Human Perception and Performance, 29(1), 92-105. doi: 10.1037/0096-1523.29.1.92

Swainson, R., \& Martin, D. (2013). Covert judgements are sufficient to trigger subsequent task-switching costs. Psychological Research, 77(4), 434-448. doi:10.1007/s00426-0120448-6; 10.1007/s00426-012-0448-6

Vandierendonck, A., Liefooghe, B., \& Verbruggen, F. (2010). Task switching: Interplay of reconfiguration and interference control. Psychological Bulletin, 136(4), 601-626. doi:10.1037/a0019791

Verbruggen, F., Liefooghe, B., \& Vandierendonck, A. (2006). Selective stopping in task switching: The role of response selection and response execution. Experimental Psychology, 53(1), 48-57. doi: 10.1027/1618-3169.53.1.48 


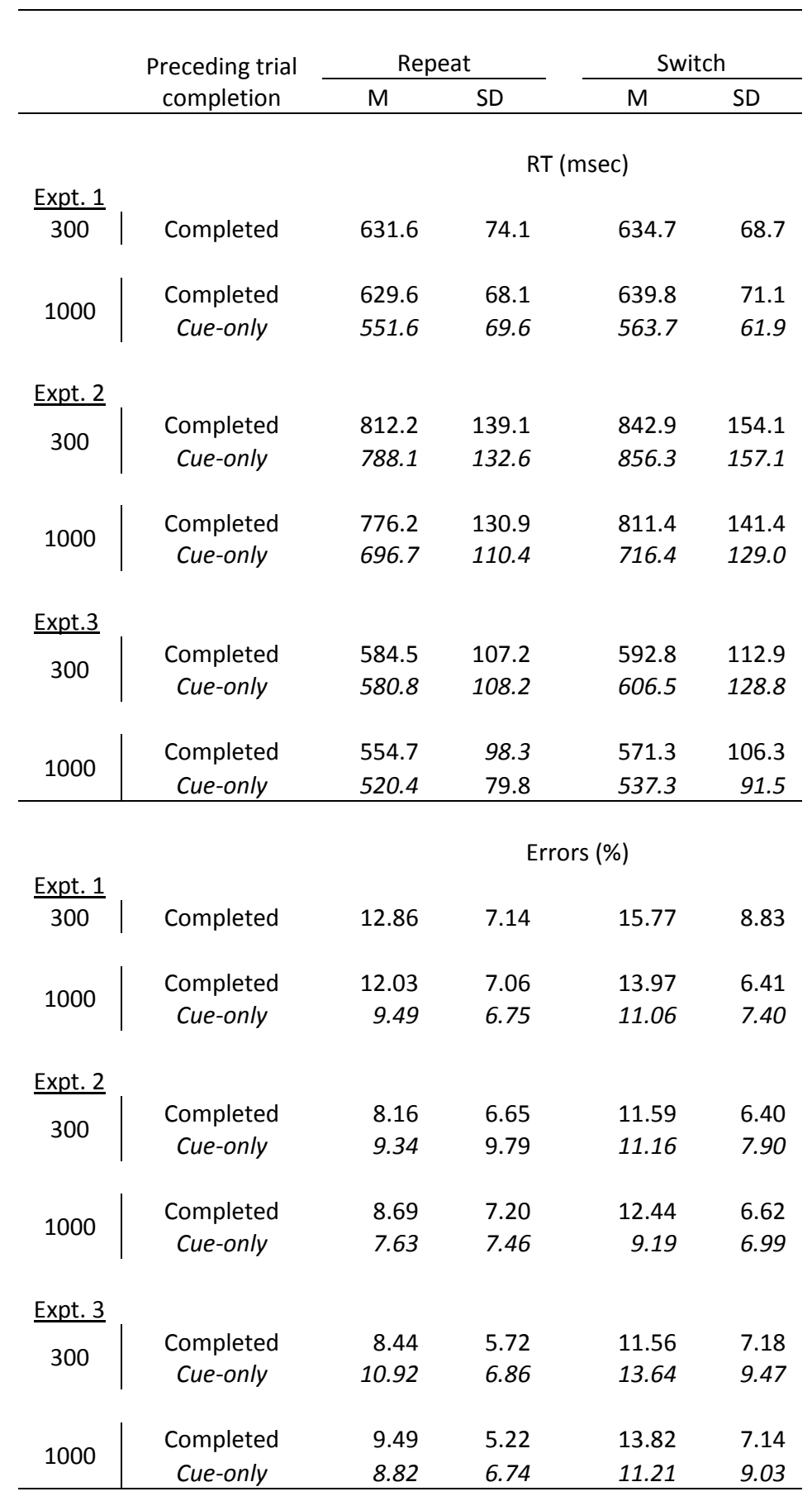

Table 1 Means (M) and standard deviations (SD) of RTs and error percentages on repeat and switch trials in each experiment by preparation interval (300 msec; $1000 \mathrm{msec})$ and preceding trial completion. Data from trials following cue-only trials are shown in italics. 
Experiment 1

Completed

(300 msec

preparation)
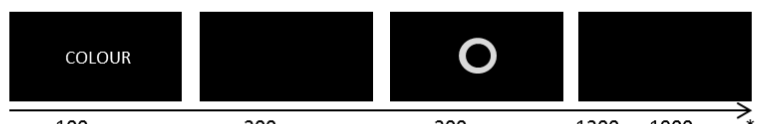

$100 \mathrm{msec}$

$200 \mathrm{msec}$

$200 \mathrm{msec}$

1200 or $1900 \mathrm{msec}^{*}$

Completed

(1000 msec

preparation)
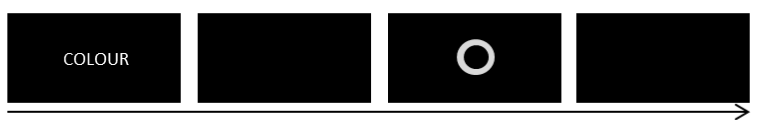

$100 \mathrm{msec}$

$900 \mathrm{msec}$

$200 \mathrm{msec}$

1200 or $1900 \mathrm{msec}^{*}$

Cue-only

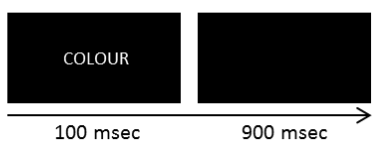

Experiment 2

Completed
(300 msec

(300 msec
preparation)

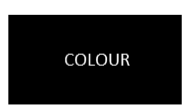

$100 \mathrm{msec}$

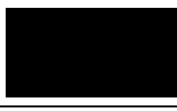

$200 \mathrm{msec}$

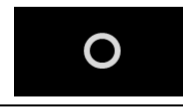

$200 \mathrm{msec}$

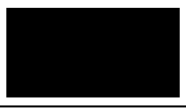

until response 600 or $1300 \mathrm{msec}^{* *}$

Completed
(1000 msec

preparation)
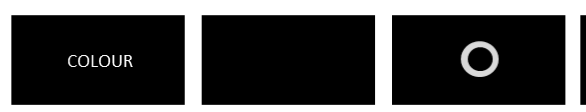

$200 \mathrm{msec}$

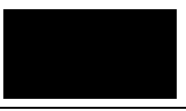

until response 600 or $1300 \mathrm{msec***}$

Cue-only

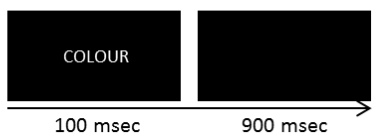

Experiment 3

Completed

(300 msec

preparation)

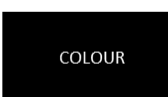

$100 \mathrm{msec}$
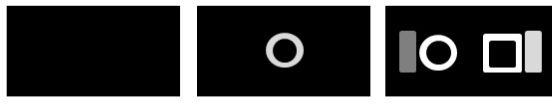

$200 \mathrm{msec}$

until response 600 or $1300 \mathrm{msec}^{* *}$

Completed

(1000 msec

preparation)

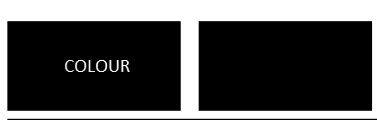

$100 \mathrm{msec}$

$900 \mathrm{msec}$

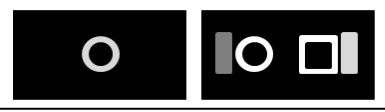

$200 \mathrm{msec}$

until response 600 or $1300 \mathrm{msec**}$

Cue-only

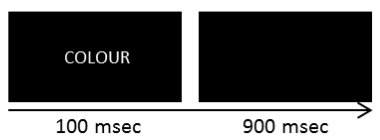

Fig. 1 Trial events and timings in each experiment. *In Experiment 1, the final blank screen was shown for $1200 \mathrm{msec}$ prior to a long preparation interval $(1000 \mathrm{msec})$ trial and 1900 msec prior to a short preparation interval $(300 \mathrm{msec})$ trial such that total trial length for completed trials was fixed at 2400 msec. The time-limit for responding in Expt. 1 was 1200 msec from target onset. **In Experiments $2 \& 3$, the final blank screen (response-cue interval) was shown for $600 \mathrm{msec}$ prior to a long preparation interval $(1000 \mathrm{msec})$ trial and $1300 \mathrm{msec}$ prior to a short preparation interval $(300 \mathrm{msec})$ trial such that total response-target interval was fixed at $1600 \mathrm{msec}$. There was no time-limit for responding in Expts. 2 \& 3. Grey bars represent two of the four possible colours (green, red, blue, yellow). 


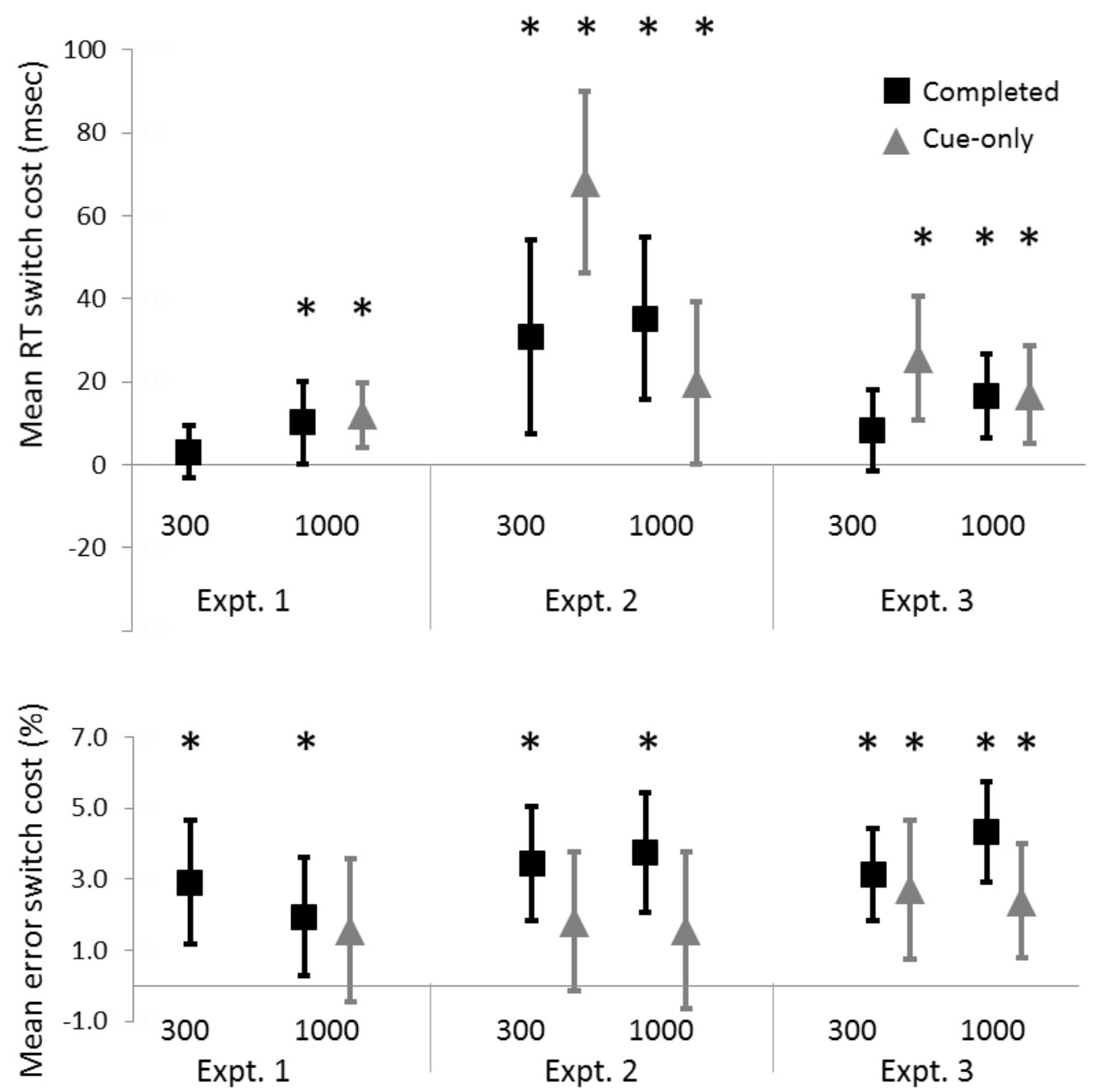

Fig. 2 Mean RT and percentage error switch costs (switch - repeat) in each experiment by preparation interval (300 $\mathrm{msec} ; 1000 \mathrm{msec})$ and preceding trial completion. Error bars show 95\% CI. Asterisks indicate switch costs that are significantly greater than 0 msec $(\mathrm{p}<0.05)$. 